\title{
Usefulness of the Medical Research Council (MRC) dyspnoea scale as a measure of disability in patients with chronic obstructive pulmonary disease
}

J C Bestall, E A Paul, R Garrod, R Garnham, P W Jones, J A Wedzicha

\begin{abstract}
Background-Methods of classifying chronic obstructive pulmonary disease (COPD) depend largely upon spirometric measurements but disability is only weakly related to measurements of lung function. With the increased use of pulmonary rehabilitation, a need has been identified for a simple and standardised method of categorising disability in COPD. This study examined the validity of the Medical Research Council (MRC) dyspnoea scale for this purpose.

Methods-One hundred patients with COPD were recruited from an outpatient pulmonary rehabilitation programme. Assessments included the MRC dyspnoea scale, spirometric tests, blood gas tensions, a shuttle walking test, and Borg scores for perceived breathlessness before and after exercise. Health status was assessed using the St George's Respiratory Questionnaire (SGRQ) and Chronic Respiratory Questionnaire (CRQ). The Nottingham Extended Activities of Daily Living (EADL) score and Hospital Anxiety and Depression (HAD) score were also measured.
\end{abstract}

Results-Of the patients studied, 32 were classified as having MRC grade 3 dyspnoea, 34 MRC grade 4 dyspnoea, and 34 MRC grade 5 dyspnoea. Patients with MRC grades 1 and 2 dyspnoea were not included in the study. There was a significant association between MRC grade and shuttle distance, SGRQ and CRQ scores, mood state and EADL. Forced expiratory volume in one second $\left(\mathrm{FEV}_{1}\right)$ was not associated with MRC grade. Multiple logistic regression showed that the determinants of disability appeared to vary with the level of disability. Between MRC grades 3 and 4 the significant covariates were exercise performance, SGRQ and depression score, whilst between grades 4 and 5 exercise performance and age were the major determinants.

Conclusions-The MRC dyspnoea scale is a simple and valid method of categorising patients with COPD in terms of their disability that could be used to complement FEV $_{1}$ in the classification of COPD severity.

(Thorax 1999;54:581-586)

Keywords: MRC dyspnoea scale; chronic obstructive pulmonary disease; dyspnoea
Patients with chronic obstructive pulmonary disease (COPD) experience a wide variation in their level of disability. Pulmonary rehabilitation, designed to improve exercise performance and quality of life and to reduce disability, is emerging as an important treatment modality in this disease. Current guidelines define the severity of COPD in terms of the level of forced expiratory volume in one second $\left(\mathrm{FEV}_{1}\right)$, but the correlation between airways obstruction and exercise performance is modest. ${ }^{12}$ Health status measurements such as that provided by the St George's Respiratory Questionnaire (SGRQ) and the Chronic Respiratory Questionnaire (CRQ) provide well validated measurements of disability and handicap due to COPD, but these are complex to administer and score. ${ }^{34}$ There is a need for a simple and standardised method of scoring disability that will allow patients and patient populations to be categorised in the manner analogous to the New York Heart Association grading for disability due to heart failure.

The Medical Research Council (MRC) dyspnoea scale has been in use for many years for grading the effect of breathlessness on daily activities. ${ }^{5}$ This scale actually measures perceived respiratory disability, the WHO definition of disability being "any restriction or lack of ability to perform an activity in the manner or within the range considered normal for a human being". The MRC dyspnoea scale is simple to administer as it allows the patients to indicate the extent to which their breathlessness affects their mobility. Whilst there is a well established relationship between MRC dyspnoea grade and walking test performance, ${ }^{6}$ there has been no formal assessment of the categories of breathlessness used in the MRC scale and other measures of impairment, disability, and handicap. In this study we stratified patients with the MRC dyspnoea scale and then tested for differences in lung function, activities of daily living, health status, and exercise tolerance between patients according to the dyspnoea grade. The study was confined to patients with some limitation of activity due to breathlessness during daily life (MRC grade 3 and above). Patients with levels of dyspnoea below this (grade 1: breathlessness on strenuous exercise, grade 2: hurrying on the level or up a slight hill) were not included in this study since we wished to explore the range of disability in patients who might be considered for pulmonary rehabilitation. Few patients with mild levels of self-reported dyspnoea on daily 
life would currently be referred to such programmes.

\section{Methods}

PATIENTS

One hundred and thirty eight patients (70 men) of median age 70 years (range 44-81) with stable severe COPD were recruited sequentially from the respiratory outpatient clinic at The London Chest Hospital over a period of 16 months. Inclusion criteria were a clinical diagnosis of COPD and a requirement that patients were in a clinically stable condition with no exacerbation for three weeks prior to assessment. Patients were included on the pulmonary rehabilitation programme if they selected grades 3, 4, or 5 from the MRC dyspnoea scale since this corresponds to moderate to severe disability due to dyspnoea. Patients who selected grades 1 or 2 were excluded since this corresponds to mild disability due to dyspnoea and these patients were not recruited onto the rehabilitation programme. Other exclusion criteria were the presence of any other disorder that would prevent the patient from being able to complete a walking test or an inability to complete questionnaires either verbally or by selfcompletion. Written informed consent was obtained from each participant and the study protocol was approved by the East London \& City Health Authority Research ethics committee.

Of the 138 patients approached, 10 declined to take part in the study leaving 128 to be stratified according to disability using the MRC dyspnoea scale. Of these, 126 patients were assessed as MRC grades 3, 4, or 5 and two were excluded because they were MRC grade 2 . The number of patients in each MRC grade was as follows: grade $3=32$ patients, grade $4=$ 34 patients, and grade $5=66$ patients. To provide equal numbers of subjects at each grade a computer generated random sample of 34 patients was selected from the 66 patients with MRC grade 5 dyspnoea. This study was therefore concerned with a total of 100 patients: 32 with MRC grade 3 dyspnoea, 34 with grade 4, and 34 with grade 5 dyspnoea.

MRC DYSPNOEA SCALE

Patients were asked about their perceived breathlessness and were then classified into MRC dyspnoea grades 3, 4, or 5 according to how they perceived their disability. The MRC dyspnoea scale is a questionnaire that consists of five statements about perceived breathlessness: grade 1, "I only get breathless with strenuous exercise"; grade 2, "I get short of breath when hurrying on the level or up a slight hill"; grade 3, "I walk slower than people of the same age on the level because of breathlessness or have to stop for breath when walking at my own pace on the level"; grade 4, "I stop for breath after walking 100 yards or after a few minutes on the level"; grade 5, " I am too breathless to leave the house". Patients selected the grade that applied to them. Those who graded themselves in MRC grades 3, 4, or 5 were entered into the study as these levels would correspond to moderate to severely disabling COPD. Patients with grades 1 and 2 dyspnoea were excluded from the study.

\section{LUNG FUNCTION PARAMETERS}

Spirometric parameters $\left(\mathrm{FEV}_{1}, \mathrm{FVC}\right)$ were measured at the first assessment using a rolling seal spirometer (PK Morgan Ltd, Rainham, Essex, UK). Predicted values were calculated using ECCS reference values. ${ }^{7}$ Spirometric values were assessed before and after the administration of a bronchodilator $(800 \mu \mathrm{g}$ salbutamol) with a period of 20 minutes after administration of bronchodilator. Resting blood gas tensions (on room air) were obtained from ear lobes ${ }^{8}$ and analysed immediately.

EXERCISE PERFORMANCE AND BREATHLESSNESS

Exercise performance was evaluated using the shuttle walking test which is an externally paced maximal exercise test. This was performed in a gymnasium between two cones placed $9 \mathrm{~m}$ apart (complete distance after turning $=10 \mathrm{~m}$ ) using standard instructions as described previously. ${ }^{9}$ Patients were played the instructions from the shuttle walking test tape cassette. They were required to walk the $10 \mathrm{~m}$ length at different speeds as indicated by bleeps on the cassette; speed was increased by a small increment after each minute. The end point of the test was determined by the patient when he/she became too breathless to maintain the required speed. No encouragement was given during the test. Learning effects are reported to be minimal after two repeated walking tests ${ }^{10}$ so the patients each performed one practice shuttle walking test followed by a second after a resting time of 30 minutes. Borg scores for perceived breathlessness ${ }^{11}$ were measured before and after the shuttle walking test with a score of zero being no breathlessness at all and a score of 10 being maximal breathlessness.

\section{HEALTH STATUS MEASUREMENT}

Health status was assessed by means of the SGRQ and the CRQ. The SGRQ has been shown to be a valid measure of impaired health in COPD, to have adequate reliability, ${ }^{3}$ and to be sensitive to change over time. ${ }^{12}$ It consists of 50 items with 76 weighted responses and has three component scores: Symptoms, Activity (the daily tasks that patients can perform such as stair climbing, dressing, shopping and socialising), and Impacts (the impact of the illness such as being embarrassed in public whilst coughing or perceiving illness as being a nuisance to family and friends). A total score is calculated from all three components. The scoring range for the components and total score is $0-100$ with a score of 100 indicating maximum disability.

The CRQ also assesses health status and was designed for measurement of changes within individuals. ${ }^{4}$ It consists of four component scores (Dyspnoea, Fatigue, Emotional Function, and Mastery) and is measured on a seven point Likert scale. These components can be combined to provide a total score from 20 to 140. Patients are asked to comment on how they have felt over the last two weeks. For the 
Table 1 Questionnaire scores related to MRC breathlessness score $(n=100)$

\begin{tabular}{llllll}
\hline Variable & $\begin{array}{l}\text { MRC grade } 3 \\
(n=32)\end{array}$ & $\begin{array}{l}\text { MRC grade } 4 \\
(n=34)\end{array}$ & $\begin{array}{l}\text { MRC grade 5 } \\
(n=34)\end{array}$ & ANOVA (F) & $p$ value \\
\hline SGRQ (Activity) & $60.5(17.9)$ & $77.6(15.9)$ & $78.8(16.0)$ & 12.3 & $<0.0001$ \\
SGRQ (Impacts) & $31.3(13.7)$ & $47.7(15.0)$ & $54.6(15.6)$ & 21.3 & $<0.0001$ \\
SGRQ (Symptoms) & $61.6(16.9)$ & $72.4(15.4)$ & $72.1(14.5)$ & 5.0 & $<0.009$ \\
CRQ (Dyspnoea) & $15.8(5.9)$ & $13.1(3.5)$ & $14.7(4.8)$ & 2.8 & $<.07$ \\
CRQ (Fatigue) & $17.5(5.1)$ & $13.3(4.6)$ & $12.4(4.7)$ & 10.6 & $<0.0001$ \\
CRQ (Emotional function) & $34.8(9.6)$ & $29.6(9.1)$ & $27.8(9.5)$ & 4.8 & $<0.0001$ \\
CRQ (Mastery) & $21.3(4.8)$ & $14.7(5.1)$ & $14.6(5.7)$ & 17.8 & $<0.0001$ \\
CRQ (Total) & $89.4(17.9)$ & $70.7(15.6)$ & $70.0(20.5)$ & 12.4 & $<0.008$ \\
HAD (Anxiety) & $4.7(3.6)$ & $7.7(4.3)$ & $7.7(4.9)$ & 5.1 & $<0.0001$ \\
HAD (Depression) & $3.5(1.9)$ & $6.2(3.0)$ & $7.1(3.2)$ & 15.4 & Kruskal-Wallis $(H)$ \\
Borg (before exercise) & $0.5(0-2)$ & $1.0(0-4)$ & $1.0(0-5)$ & 10.5 & $<0.005$ \\
Borg (after exercise) & $3(1-6)$ & $4(1-7)$ & $4(2-10)$ & 7.1 & $<0.03$ \\
\hline
\end{tabular}

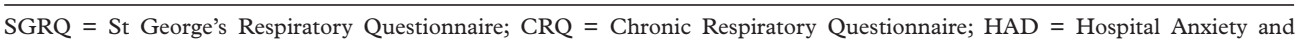
Depression score; EADL $=$ Extended Activities of Daily Living score.

${ }^{\star} \mathrm{CRQ}$ total is a sum of component scores.

Dyspnoea component each patient selected five activities that made them feel breathless and which were important in their day to day life. They then indicated how breathless they were when doing these activities. The Fatigue component measured how tired the patient was, the Emotional Function component measured how anxious or depressed they were, and the Mastery component addressed the confidence of the patients in dealing with their illness.

MOOD STATE AND DAILY ACTIVITY

The Hospital Anxiety and Depression (HAD) score was used to assess mood state. This questionnaire consists of 14 items which produce separate scores for anxiety and depression. ${ }^{13}$ The scores range from 0 to 21 and a score of 10 or more indicates a clinically significant case of anxiety or depression.

Activities of daily living were measured using the Nottingham Extended Activities of Daily Living (EADL) scale which is an instrument with 22 items which record the number of activities (from 22 listed) that the patient has engaged in during the previous week. ${ }^{14}$ Scores range from 0 to 22 with a score of 22 indicating a high level of activity.

These questionnaires were completed at one sitting and each patient completed the questionnaires in the same order. Most patients were able to complete the questionnaires unaided; those who were unable to complete the questionnaires themselves due to shaky hands, inability to read, or poor eyesight had the questionnaires read out to them in the exact format in which they were set out. Nondirective guidance was given on the few occasions when patients had queries on how to answer questions.

\section{STATISTICAL ANALYSIS}

Lung function, blood gas data, HAD score, and health status measures had normal distributions so differences between patients with MRC grades 3, 4, and 5 dyspnoea were compared using analysis of variance (ANOVA). The shuttle walking distance was not normally distributed between groups and was log transformed to provide a normal distribution. For those variables showing a significant difference on ANOVA, Fisher's PLSD was used to deter- mine where the difference between the three disability grades lay. The EADL and Borg scores were not normally distributed and could not be normalised using any form of transformation and so comparisons were made using the Kruskal-Wallis test. Comparison between grades 3 and 4 and grades 4 and 5 were made using the Mann-Whitney test. Statistical significance for all analyses was accepted at a level of $\mathrm{p}<0.05$.

If a number of variables showed a significant association with MRC dyspnoea grade in the univariate analysis, a multivariate analysis was planned to test which of these associations was maintained after adjustment for covariance between variables. We hypothesised that the factors associated with different MRC dyspnoea grades may vary with the degree of disability. For this reason, and because it is difficult to carry out multivariate analysis using a categorical dependent variable, we carried out two separate analyses. One compared MRC grade 3 with MRC grade 4, the other compared MRC grade 4 with MRC grade 5 . We used multiple logistic regression since we were testing for associations between a number of continuous variables and a binary statethat is, the state of belonging to one or other MRC dyspnoea grade. The covariates were entered into the logistic regression using a stepwise backwards technique. Logistic regression analysis predicts the odds (log odds ratio) of an association between a variable and, in this case, one or other category of MRC dyspnoea grade. For example, when comparing differences in the size of a given variable between patients in MRC grades 4 and 5, an odds ratio of 1.0 indicates that the variable was of the same magnitude in both groups. If the $95 \%$ confidence interval for the odds ratio does not include 1.0, that variable was significantly different between the two MRC grades at $\mathrm{p}<0.05$.

\section{Results}

One hundred patients (55 men) of median age 70 years (range $44-86$ ) were studied. There were 32 patients with MRC grade 3 dyspnoea, 34 patients with MRC grade 4 and 34 patients with MRC grade 5 dyspnoea. Measures of lung function, exercise tolerance, health status, 
Table 2 Age and physiological parameters in 100 patients with COPD categorised according to MRC dyspnoea grade

\begin{tabular}{llllll}
\hline Variable & $\begin{array}{l}\text { MRC grade } 3 \\
(n=32)\end{array}$ & $\begin{array}{l}\text { MRC grade 4 } \\
(n=34)\end{array}$ & $\begin{array}{l}\text { MRC grade 5 } \\
(n=34)\end{array}$ & $\begin{array}{l}\text { ANOVA } \\
(F)\end{array}$ & p value \\
\hline Age (years) & $69.3(7.2)$ & $67.5(8.3)$ & $73.2(5.5)$ & 5.66 & $<0.005$ \\
FEV $_{1}$ prebronchodilator $(1)$ & $0.99(0.38)$ & $0.91(0.37)$ & $0.82(0.36)$ & 1.81 & 0.2 \\
FEV $_{1}(\%$ predicted) & $40.8(14.6)$ & $36.5(15.6)$ & $37.0(15.1)$ & 0.79 & 0.5 \\
$\Delta \mathrm{FEV}_{1}(\%$ predicted) & $4.2(4.4)$ & $2.3(3.3)$ & $2.6(3.1)$ & 2.50 & 0.09 \\
$\mathrm{FVC}_{(1)}$ & $2.57(0.75)$ & $2.37(0.68)$ & $1.92(0.73)$ & 6.83 & $<0.002$ \\
$\mathrm{PaCO}_{2}(\mathrm{kPa})$ & $5.90(0.67)$ & $5.70(0.75)$ & $5.96(0.77)$ & 0.67 & 0.5 \\
$\mathrm{PaO}_{2}(\mathrm{kPa})$ & $8.88(1.09)$ & $8.65(0.76)$ & $8.63(1.45)$ & 0.49 & 0.6 \\
$\mathrm{pH}$ & $7.41(0.02)$ & $7.41(0.07)$ & $7.43(0.02)$ & 1.58 & 0.2 \\
\hline
\end{tabular}

$\mathrm{FEV}_{1}=$ forced expiratory volume in one second $\mathrm{FVC}=$ forced vital capacity; $\mathrm{PaCO}_{2}, \mathrm{PaO}_{2}=$ arterial carbon dioxide and oxygen tensions.

Table 3 Mean (95\% CI) differences between MRC dyspnoea grade determined by post hoc tests (Fisher's PLSD)

\begin{tabular}{lllll}
\hline Variable & MRC 3 vs MRC 4 & pvalue & MRC 4 vs MRC 5 & p value \\
\hline SGRQ & $17.1(8.7$ to 25.4$)$ & $<0.0001$ & & \\
$\quad$ Activity & $16.4(9.3$ to 23.4$)$ & $<0.0001$ & \\
Impact & $-4.2(-6.5$ to -1.8$)$ & $<0.0006$ & & \\
CRQ & $-6.6(-8.9$ to -4.1$)$ & $<0.0001$ & & \\
Fatigue & $-18.7(-26.9$ to -10.5$)$ & $<0.0001$ & & \\
Mastery & & $<0.006$ & & \\
$\quad$ Total & $3.0(0.9$ to 4.9$)$ & $<0.0001$ & & \\
HAD & $2.7(1.4$ to 3.9$)$ & & $-0.7(2.2$ to 9.1$)$ & $<0.001$ \\
Anxiety & & & $-0.4(-0.8$ to 0.09$)$ & $<0.02$ \\
$\quad$ Depression & & & \\
Age & & & \\
FVC & & &
\end{tabular}

SGRQ = St George's Respiratory Questionnaire; CRQ = Chronic Respiratory Questionnaire; $\mathrm{HAD}=$ Hospital Anxiety and Depression score; FVC $=$ forced vital capacity.

mood state, daily activity, and breathlessness were analysed across the three MRC groups.

LUNG FUNCTION AND MEDICAL HISTORY

The patients with MRC grade 5 dyspnoea were older than those with grades 3 and 4 dyspnoea and their FVC was lower than that measured for patients in grades 3 and 4 (tables 2 and 3). There were no differences between patients with the three MRC dyspnoea grades in terms of $\mathrm{FEV}_{1}$ or blood gas measurements.

Of the 100 patients, $97 \%$ had a smoking history and only $3 \%$ had never smoked; $27 \%$ were current smokers. Median (range) pack years for each MRC grade was $33(0-200)$ years for patients with grade 3 dyspnoea, 41 (0-120) years for those with grade 4 dyspnoea, and 43 (1-150) years for those with grade 5 dyspnoea $(\mathrm{p}=0.64)$.

All patients were receiving salbutamol. The main difference in treatment was that the patients with grade 4 and 5 dyspnoea were more likely to use a nebuliser for bronchodilators or anticholinergic drugs than those with grade 3 dyspnoea $(\mathrm{n}=6,16$, and 26 for grades 3,4 , and 5, respectively; $\mathrm{p}<0.0001, \chi^{2}$ test). In addition, more patients with grade 5 dyspnoea were on long term oxygen therapy $(n=15)$ than with grade $3(n=2)$ or grade $4(n=6)$ dyspnoea $\left(\mathrm{p}<0.002, \chi^{2}\right.$ test $)$.

EXERCISE TOLERANCE AND BREATHLESSNESS

The distance covered in the shuttle walking test decreased significantly as the MRC grade increased (fig 1). Assessment of breathlessness using the Borg score showed that, even though the level of perceived breathlessness at rest was low in each grade, there were significant differences between MRC grades. Perceived breathlessness after exercise was also significantly different between grades (table 1). Most of the patients $(n=93)$ indicated moderate to severe breathlessness as a reason for stopping on the walking test; the remaining seven indicated that their breathlessness was very slight or slight after the walking test. Other reasons for stopping included general fatigue $(n=1)$, leg fatigue $(n=5)$, and unwillingness to walk faster $(\mathrm{n}=1)$.

\section{HEALTH STATUS}

The results of the SGRQ and the CRQ are shown in fig 1 and table 1 . Analysis of variance showed significant differences between MRC grades for all SGRQ scores. Post hoc tests (table 3) revealed that the largest difference occurred between grades 3 and 4, while patients with grades 4 and 5 dyspnoea had similar scores. The CRQ behaved in the same way as the SGRQ. Differences in scores for Fatigue, Emotional Function, Mastery, and Total were greatest between grades 3 and 4 . There were no significant differences in the CRQ Dyspnoea scores between the different MRC grades.

\section{MOOD STATE AND DAILY ACTIVITY}

There were differences in anxiety and depression scores between the three groups (table 1). Post hoc tests (table 3 ) revealed that the largest differences occurred between grades 3 and 4, in a pattern similar to that shown by the measures of health status.

Daily activity assessed by the Extended Activities of Daily Living (EADL) score was significantly different between the groups. The median scores (fig 1 ) revealed that there was a progressive decline in ability to perform daily activity as disability level, indicated by the MRC scale, increased.

FACTORS ASSOCIATED WITH MRC GRADES Variables that were both normally distributed and were significantly different between MRC grades were considered for inclusion in stepwise backward logistic regression analysis. One regression examined the factors that were different between MRC grades 3 and 4, the second examined differences between MRC grades 4 and 5 . The factors that were examined were age, log transformed exercise performance, health status, and mood state. For those factors where there was more than one measured variable, the variable chosen to be representative was the one that had the highest level of association with MRC grade in the ANOVA. For the health status domain the SGRQ total score was used and for mood state depression was used. Spirometric measures were not included in these multiple regressions because the univariate analysis showed that even the FVC only just achieved the threshold of significance for differences between MRC grades 4 and 5 and was not significantly different between grades 3 and 4 (table 3 ). The EADL score could not be included in the logistic regressions because it was not possible to find a transformation function that could normalise these data.

These multivariate regressions (table 4) showed that exercise tolerance, health status, 

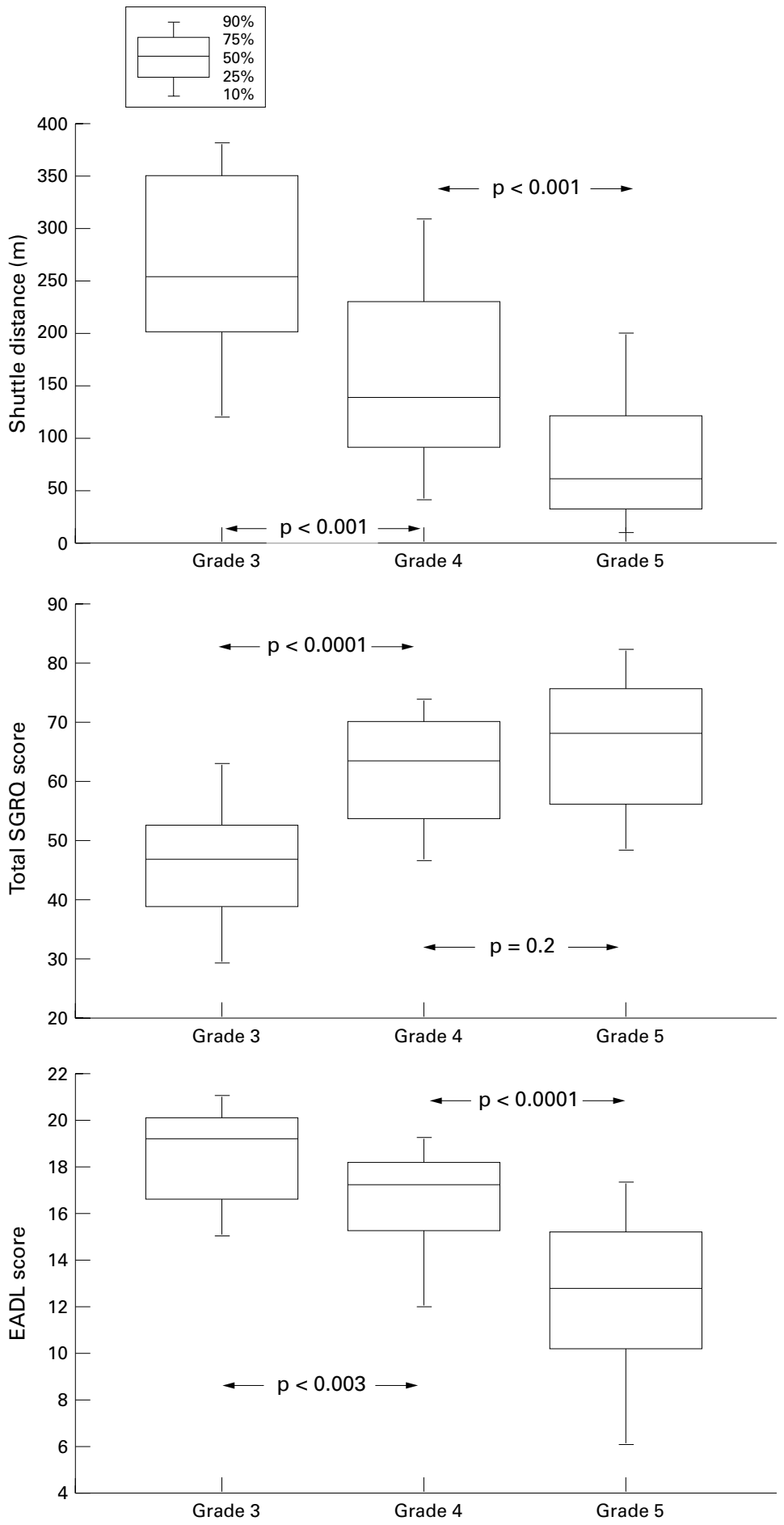

Figure 1 Shuttle walking distance, St George's Respiratory Questionnaire (SGRQ) total score, and Nottingham Extended Activities of Daily Living (EADL) score for patients with MRC dyspnoea grades 3, 4, and 5.

Table 4 Regression of variables between MRC grades: odds ratios, 95\% confidence intervals and level of significance

\begin{tabular}{llll}
\hline Outcome variable & Explanatory variable & Odds ratio $(95 \%$ CI) & p value \\
\hline MRC3 vs MRC 4 & Shuttle & $0.33(0.001$ to 0.613$)$ & $<0.02$ \\
& SGRQ total & $1.09(1.06$ to 1.39$)$ & $<0.008$ \\
MRC 4 vs MRC 5 & HAD depression & $1.42(1.37$ to 1.95$)$ & $<0.03$ \\
& Shuttle & $0.11(0.02$ to 0.6$)$ & $<0.01$ \\
& Age & $1.13(1.02$ to 1.24$)$ & $<0.02$ \\
\hline
\end{tabular}

and depression were all significantly different between patients with MRC grades 3 and 4 dyspnoea. Age and exercise tolerance were significantly different between patients with grades 4 and 5 dyspnoea.

\section{Discussion}

The purpose of this study was to determine the level of association between disability due to breathlessness categorised by the MRC dyspnoea scale and other variables used to measure the severity and impact of COPD. The factors that determined whether a patient fell within MRC grade 3 or 4 were exercise tolerance, health status, and mood state while age and exercise tolerance appeared to be principal determinants between grades 4 and 5 . In addition, there was a highly significant worsening of activities of daily living between patients with MRC grades 4 and 5 dyspnoea. It therefore appears that the correlates of disability due to breathlessness may vary with the level of disability. We believe that this is the first time this has been demonstrated.

The patients with MRC grade 5 dyspnoea were, on average, over 70 years of age whereas patients with grades 3 and 4 dyspnoea were younger. The reason for this age related factor is not clear since it is not explicable solely in terms of exercise limitation as age and shuttle distance were both significant covariates in a multiple logistic regression between grades 4 and 5. Furthermore, age was not correlated with any other variable studied.

It is particularly noteworthy that the $\mathrm{FEV}_{1}$ did not relate to disability as measured using the MRC scale. The mean $\mathrm{FEV}_{1}$ was less than one litre in all three groups and, although $\mathrm{FEV}_{1}$ was lowest with the highest MRC grade, this was not significant. One possible reason for this lack of variation is that the range of differences in $\mathrm{FEV}_{1}$ across the groups was very small. These findings suggest that, in patients with this degree of airway obstruction, any given $\mathrm{FEV}_{1}$ may be associated with a wide range of disability. Clearly, measurements of disability are complementary to those obtained by spirometric testing.

Performance on the shuttle walking test was related to the level of disability and the mean scores clearly deteriorated as disability increased across the MRC groups, although there was an overlap in shuttle measurements between the three groups. The overlap in shuttle distance between grades may be due to several factors including change in functional residual capacity, dynamic hyperinflation of the lungs during exercise, degree of muscle wasting or "deconditioning", as well as the patients' perception of their decline.

There were large differences in scores for health status and mood state between patients with MRC grades 3 and 4 dyspnoea but not between those with grades 4 and 5 dyspnoea. The reasons for this are not entirely clear. The same pattern was seen with the SGRQ and CRQ, so this does not appear to be a feature of a particular questionnaire. Furthermore, it does not appear to be due to a "floor effect"that is, it is not because the patients had 
reached the extreme end of the scoring range. However, there is the possibility that such questionnaires, with their emphasis on activity and social function, may provide discrimination between levels of disability in patients who can leave the home but fail to register levels of worsening disability in people who have deteriorated to the point at which they are effectively housebound. Support for this conclusion comes from the observation that the EADL scale, which focuses on activities within the home, was significantly different both between grades 3 and 4 and between grades 4 and 5 .

One limitation of this study concerns the range of disability studied. We restricted this to patients with MRC grade 3 dyspnoea and above because we felt that such patients were eligible for pulmonary rehabilitation programmes and our concern was to validate a simple method for defining the level of perceived disability in COPD patients in such a setting. We argued that, since rehabilitation is designed to improve exercise performance and reduce disability, it was likely that most patients identified as potentially benefiting from a rehabilitation programme would have significant disability in their daily lives. This approach restricted our test of the usefulness of the MRC scale to patients at the more severe end of the spectrum, but it should be noted that fewer than $2 \%$ of a consecutively approached sample of patients with COPD recruited from a hospital outpatient population had an MRC dyspnoea grade of 2 or lower.

In conclusion, this study has shown that the MRC dyspnoea scale provides a simple and valid method of categorising patients in terms of their disability due to COPD. We suggest that careful consideration should be given to the use of this scale in any system used to classify COPD, and that the MRC grade should be recorded in all descriptions of COPD populations.

We are grateful to the NHS Research and Development Programme on Physical and Complex Disability for funding this study. We would also like to thank Leonette John and her staff at the Respiratory Function Unit for help with measurements.

1 Morgan AD, Peck DF, Buchannan DR, et al. Effect of attitudes and beliefs on exercise tolerance in chronic bronchitis. BMF 1983;286:171-3.

2 Killian KJ, Leblanc P, Martin DH, et al. Exercise capacity and ventilatory, circulatory and symptom limitation in patients with chronic airflow obstruction. Am Rev Respir Dis 1992;146:935-40.

3 Jones PW, Quirk FH, Baveystock CM, et al. A self-complete measure of health status for chronic airflow limitation: the St George's Respiratory Questionnaire. Am Rev Respir Dis 1992;145:1321-7.

4 Guyatt GH, Berman LB, Townsend $\mathrm{M}$, et al. A measure of quality of life for clinical trials in chronic lung disease. Thorax $1987 ; 42: 773-8$.

5 Fletcher CM (Chairman). Standardised questionnaire on respiratory symptoms: a statement prepared and approved by the MRC Committee on the Aetiology of Chronic Bronchitis (MRC breathlessness score). BMF 1960;2: 1665.

6 McGavin CR, Artvinli M, Naoe H, et al. Dyspnoea, disability and distance walked: comparison of estimates of exercise performance in respiratory disease. BMf 1978;2: 241-3.

7 Quanjer P, Tammeling GJ, Cotes JE, et al. Standardised lung function testing: lung volumes and forced ventilatory flows. Eur Respir f 1993;6(Suppl 16):5-40.

8 Pitkin AD, Roberts CM, Wedzicha JA. Arterialised ear lobe blood gas analysis: an underused technique. Thorax 1994;49:364-6.

9 Singh SJ, Morgan MDL, Scott S, et al. Development of a shuttle walking test of disability in patients with chronic shuttle walking test of disability in patients with
airways obstruction. Thorax 1992;47:1019-24.

10 McGavin CR, Gupta SP, McHardy GJR. Twelve minute walking test for assessing disability in chronic bronchitis. walking test for assessi

11 Burdon GW, Juniper EF, Killian KJ, et al. The perception of breathlessness in asthma. Am Rev Respir Dis 1982;126:8258.

12 Jones PW, Bosh TK. Changes in quality of life in COPD patients treated with salmeterol. Am $\mathcal{F}$ Respir Crit Care Med 1997;155:1283-9.

13 Zigmond AS, Snaith RP. The Hospital Anxiety and Depression Scale. Acta Psychiatr Scand 1983;67:361-70.

14 Lincoln NB, Gladman JRF. The extended Activities of Daily Living Scale: a further validation. Disability $\mathcal{E}$ Rehabilitation 1992;14:41-3. 